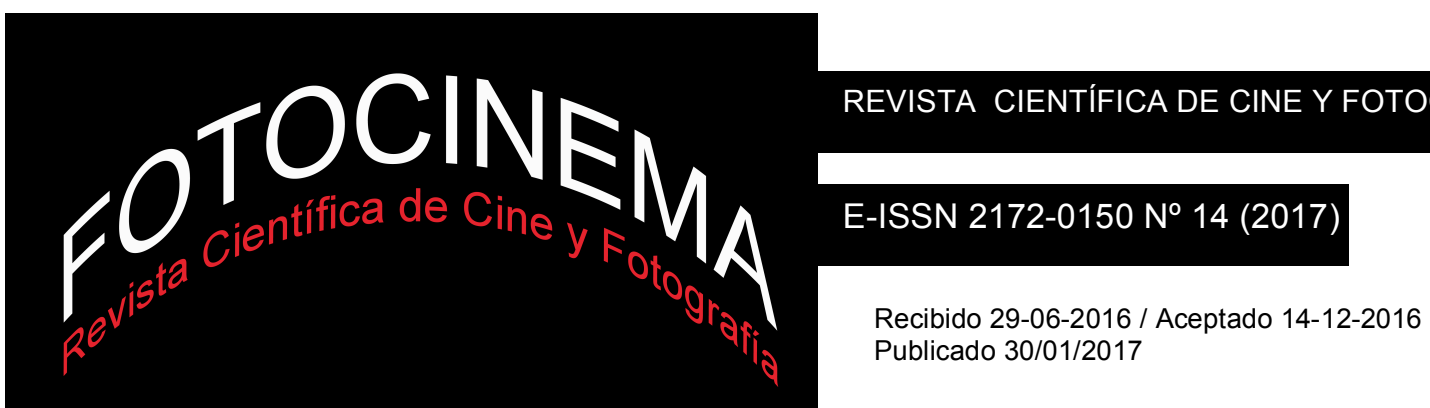

\title{
PRESENTE Y FUTURO DE LAS AYUDAS A LA INDUSTRIA CINEMATOGRÁFICA ESPAÑ̃LA
}

\section{PRESENT AND FUTURE OF GRANTS TO SPANISH FILM INDUSTRY}

Olga Heredero Díaz

Universidad Complutense de Madrid, España olga.heredero.díaz@ucm.es

Francisco Reyes Sánchez

Universidad Complutense de Madrid, España freyessa@ccinf.ucm.es

\section{Resumen:}

El descenso continuado de los principales indicadores de la industria cinematográfica española revela la crisis de un sector incapaz de hacer frente a un conjunto de problemas agravados por la subida del IVA cultural al 21\%, junto con una rebaja progresiva de las ayudas públicas estatales y autonómicas que acabó en 2015, cuando por primera vez desde 2009 se incrementaba la inversión estatal en esta partida con la implementación de un nuevo modelo de subvenciones a priori. Con el fin de aclarar la controversia sobre si las ayudas al cine tienen realmente efectos positivos, se propone la determinación de la existencia de una correlación positiva entre las cuantías anuales de las ayudas estatales a la cinematografía y los principales indicadores del estado del sector entre 2004 y 2014: número de películas españolas producidas, número de películas españolas estrenadas y total de espectadores de cine español. El análisis de los resultados obtenidos pone en cuestión las posturas que argumentan que el fortalecimiento de la industria cinematográfica española debe sustentarse en un sistema de subvenciones que contribuya a su financiación.

\begin{abstract}
:
The continued decline of the main indicators of the Spanish film industry reveals the crisis of one sector to incapable face a set of problems exacerbated by the rise of cultural VAT to $21 \%$, along with a progressive reduction of state and regional aids which it ended in 2015, when for the first time since 2009 the state investment was increased in this game with the implementation of a new grant model a priori. In order to clarify the controversy over if whether film funding actually have positive effects, determining the existence of a positive correlation between the annual amounts of state aid to the film and the main indicators of the state of the industry from 2004 and 2014: Spanish number of films produced, the number of Spanish films released and total viewers of Spanish cinema. The analysis of the results put into question the positions that argue that strengthening the Spanish film industry must be based on a system of subsidies that contribute to their funding.
\end{abstract}

Palabras clave: subvenciones; industria cinematográfica; ayudas a la cinematografía; cine español; exhibidores; digitalización.

Keywords: Grants; Film Industry; Aid to Cinematography; Spanish Cinema; Cinema Exhibitors; Digitization. 


\section{Introducción: las cifras del sector cinematográfico en España}

El descenso progresivo en los principales indicadores de la industria del cine español en los últimos años, tales como la recaudación, el número de espectadores, el total de pantallas y salas operativas o el número de sesiones por pantalla, ponen de manifiesto las dificultades de un sector afectado por la crisis económica, la piratería audiovisual, el gran peso del cine norteamericano respecto a la recaudación en taquilla, los cambios en los patrones de consumo de ocio o la subida del IVA cultural al 21\%, vigente desde el 1 de septiembre de 2012.

En cifras macroeconómicas difundidas por la Federación de Cines de España (FECE), la taquilla española representa el 10\% de la taquilla de la Unión Europea y el 15\% de sus pantallas. No obstante, estos datos se han de analizar teniendo en cuenta los proporcionados por la Encuesta de Hábitos y Prácticas Culturales 2014-2015, la última publicada, que indican que las actividades culturales más frecuentes, en términos anuales, son escuchar música $(87,2 \%)$, leer $(62,2 \%)$ e ir al cine (54\%), de forma que entre los espectáculos culturales el cine continúa siendo, con gran diferencia, el que tiene más adeptos. Esta cifra es superior en los varones y en los más jóvenes, siendo los solteros que viven en casa de sus padres $(78,4 \%)$ y las parejas con hijos menores (60,9\%), los asistentes más asiduos (MECD, 2015, p. 37). En cuanto a los géneros cinematográficos, las preferencias del público se inclinan por las comedias (29,7\%), películas de acción (19,6\%), de ciencia ficción $(11,7 \%)$, dramáticas $(8,5 \%)$ y de aventuras $(6,5 \%)$, pero sobre todo llama la atención el porcentaje del público que opta por películas infantiles $(9,2 \%)$ o de dibujos animados (3,7\%) (MECD, 2015, p. 49).

Sin embargo, según el Anuario de Estadísticas Culturales 2015 el gasto de los hogares españoles en bienes y servicios culturales registra un descenso progresivo desde el año 2007, alcanzando los 653,6 euros anuales por hogar y los 260,1 euros por persona (MECD, 2015, p. 28).

Asimismo, según el Informe Anual 2015 Cine elaborado por la empresa especializada en el control de audiencias, Rentrak Spain, el mercado cinematográfico en España en su conjunto alcanzó un total de 572,7 millones 
de euros y 94,5 millones de espectadores en 2015. Por su parte, las producciones españolas recaudaron 109 millones de euros y fueron vistas por 18 millones de espectadores el pasado año, con una cuota de mercado por encima del 19\%, la segunda mejor tras la de 2014.

Otros datos complementarios que describen la evolución del sector en España son los que aporta el Informe sobre el estado de la cultura en España. La cultura como motor de cambio 2016 de la Fundación Alternativas, que revela la concentración de espectadores y recaudación del cine español en 2014 en unos pocos títulos, ya que Ocho apellidos vascos (Emilio Martínez-Lázaro, 2014) recaudó por sí sola el $42 \%$ de la taquilla nacional. $\mathrm{Y}$ es que, según el citado informe "el porcentaje de películas españolas que no encuentran salas de exhibición ni plataformas digitales de pago aumenta de forma paralela al incremento de la producción: solo se estrenan 2 de cada 3 películas rodadas” (Álvarez \& López, 2015, p. 43-44).

En cuanto a la exhibición, desde que la reconversión azotase a las salas rurales en los años 60 hasta llegar al fin del modelo tradicional gracias a la digitalización e Internet que se augura cercano, el sector ha sobrevivido a transformaciones profundas como la aparición de las multisalas a mediados de los años 70 o las mejoras en el confort y el sonido de las salas acometidas por los exhibidores en los años 90 (García, 2015). En este sentido, se ha de señalar que el descenso año tras año en el número de pantallas ha sido continuado, así como el del número de cines, tal y como se recoge en el Anuario de la Sociedad General de Autores y Editores de las artes escénicas, musicales y audiovisuales 2015 (SGAE, 2015, p. 14). En concreto, los datos más actualizados correspondientes al Decimonoveno Censo de Salas de Cine de la Asociación para la Investigación de Medios de Comunicación (AIMC) revelan que en abril de 2016 eran 675 los locales de exhibición en España -4 menos que el año anterior-, con 3.492 pantallas activas y un total de 778.718 butacas -una media de 17 butacas por cada 1000 habitantes-.

Sorprende que más de la mitad de las salas se localicen en Cataluña (658 salas), Andalucía (con 541) y Madrid (con 485), aunque el 61,2\% de los españoles residen en un núcleo poblacional con al menos una sala de cine y 
son 75 las salas de cine existentes por cada millón de habitantes en España -18 menos que hace diez años-. El informe revela además que el 95,1\% de las salas ya han completado su digitalización y que el 26,4\% del total de los locales poseen capacidad para la exhibición de películas en 3D.

\begin{tabular}{|l|rrrr|}
\cline { 2 - 5 } \multicolumn{1}{c|}{} & \begin{tabular}{r} 
Total \\
\multicolumn{1}{c|}{}
\end{tabular} & $\begin{array}{r}\text { \% pantallas } \\
\text { pantallas }\end{array}$ & \% pantallas & Pantallas por \\
digitales & 3D & millón hab. \\
\hline ANDALUCÍA & 541 & 94.6 & 20.0 & 64.4 \\
ARAGÓN & 86 & 89.5 & 37.2 & 65.3 \\
ASTURIAS & 65 & 98.5 & 36.9 & 61.8 \\
BALEARES & 78 & 98.7 & 23.1 & 70.6 \\
CANARIAS & 141 & 90.8 & 41.1 & 67.1 \\
CANTABRIA & 39 & 89.7 & 23.1 & 66.6 \\
CASTILLA Y LEÓN & 206 & 98.5 & 37.4 & 83.3 \\
CASTILLA-LA MANCHA & 136 & 97.8 & 17.6 & 66.0 \\
CATALUÑA & 658 & 98.5 & 25.4 & 87.6 \\
COMUNIDAD VALENCIANA & 424 & 93.9 & 18.2 & 85.1 \\
EXTREMADURA & 55 & 89.1 & 34.5 & 50.3 \\
GALICIA & 172 & 92.4 & 30.8 & 62.9 \\
MADRID & 485 & 96.9 & 32.0 & 75.3 \\
MURCIA & 119 & 81.5 & 16.8 & 81.1 \\
NAVARRA & 60 & 95.0 & 18.3 & 93.7 \\
PAIS VASCO & 178 & 92.7 & 33.1 & 81.3 \\
RIOJA, LA & 41 & 100.0 & 24.4 & 129.3 \\
CEUTA Y MELILLA & 8 & 100.0 & 12.5 & 47.1 \\
\hline TOTAL & 342 & 95.1 & 26.4 & 74.9 \\
\hline
\end{tabular}

F1. Pantallas de cine por comunidades autónomas en 2016. Decimonoveno Censo de Salas de Cine (AIMC, 2016, p. 5).

Sin embargo, lo verdaderamente alarmante en relación al retroceso en la infraestructura de la exhibición española es que en más del 40\% del territorio español no existan salas de cine. Ciudades como Pontevedra (82.00o hab.), Tarragona (132.00o hab.), Jaén (115.0oo hab.), Orihuela (83.000 hab.), Avilés (83.00o hab.) o Soria (39.00o hab.) se han quedado sin salas en sus áreas urbanas en los últimos tiempos, una tendencia que aumenta conforme desciende el tamaño del núcleo poblacional, lo que se justifica porque el gasto medio por hogar en bienes y servicios culturales disminuye al descender el tamaño del municipio, tomando su valor máximo en los 
municipios con más de 100.000 habitantes según el Anuario de Estadísticas Culturales 2015 (MECD, 2015, p. 28).

La realidad actual es que las salas han de adaptarse al cine digital (un proyector cuesta entre 60.000 y 80.000 euros), a lo que hay que añadir la aplicación $3 \mathrm{D}$, lo que eleva la inversión hasta los 120.000 euros más el coste de las gafas para los espectadores -las más caras cuestan 6o euros y sirven solo para 500 proyecciones-. Un problema agravado por el hecho de que en la mayoría de los casos los exhibidores deben hacer frente en solitario a un desembolso económico que se antoja inasumible dada la falta de ayudas.

No obstante, el dato más relevante en lo que a la industria cinematográfica se refiere es el hecho de que según el Directorio Central de Empresas (DIRCE) del Instituto Nacional de Estadística (INE), las empresas cuya principal actividad económica es cultural y se circunscribe a las actividades cinematográficas, de vídeo, radio, televisión y edición musical generaron tan solo el 8,3\% de los 107.922 millones de euros procedentes de las empresas culturales en 2014 (MECD, 2015, p. 26).

\section{Marco teórico y metodología}

\subsection{La financiación de la cultura cinematográfica en España: estado actual de las ayudas a la exhibición cinematográfica}

La promoción de la cultura ocupa un papel central en los documentos centrales de la agenda 2020 de la Unión Europea entendida como un valor social capaz de promover la innovación y el emprendimiento hasta transformarse en un sector motor de la economía.

Y es que la protección y defensa de la financiación pública de la cultura posee una regulación internacional que obliga también al Estado español que la suscribe (Aranzubía, 2014, p. 82). Es el caso de la Convención sobre la protección y promoción de la Diversidad de Expresiones Culturales, aprobada por la $33^{\text {a }}$ Asamblea de la UNESCO (París, 20 de octubre de 2005), y ratificada por España en enero de 2007 (BOE de 12 de febrero de 2007). 
Asimismo, la Resolución del Consejo Europeo, de 12 de febrero de 2001, sobre las Ayudas nacionales al sector del cine y al sector audiovisual (Diario Oficial C 73 de 6 de marzo de 2001) como continuación de la Comunicación de la Comisión, de 14 de diciembre de 1999, Principios y directrices de la política comunitaria en el sector audiovisual en la era digital, incide en la necesidad de mantener y poner en marcha dispositivos nacionales de apoyo a las industrias culturales, en tanto en cuanto, los sectores cinematográfico y audiovisual europeos desempeñan una función primordial en la conservación de la diversidad cultural europea.

No obstante, las propias autoridades europeas han puesto en marcha iniciativas de refuerzo de los sectores cultural y audiovisual europeos proporcionándoles financiación, -unos 150 millones euros desde 1998- a través de los programas marco de investigación y desarrollo tecnológico. Por su parte, el Fondo Europeo de Desarrollo Regional ha asignado 3.300 millones de euros a la protección y conservación del patrimonio cultural, 2.200 millones de euros al desarrollo de infraestructuras culturales y 555 millones de euros a apoyar servicios culturales desde 2007. Además, los programas Cultura, MEDIA y MEDIA Mundus han sido recientemente sustituidos por el Programa Europa Creativa, que, con una duración de siete años subvencionará como mínimo a 250.000 artistas y profesionales de la cultura, 2.000 salas de cine, 800 películas y 4.500 traducciones editoriales. Así, la Unión Europea invertirá 1.460 millones de euros durante el período 2014-2020 en los sectores cultural y audiovisual, lo que supone un incremento del 9 \% de los presupuestos anteriores (Comisión Europea, 2014, p. 3).

Sin embargo, la Unión Europea no renuncia a incentivar la política de ayudas de sus miembros. Y es que, según el artículo 87 del Tratado de la Comunidad Europea, las ayudas destinadas a promover la cultura y la conservación del patrimonio cultural pueden considerarse compatibles con el mercado común, de forma que las ayudas nacionales son esenciales en la medida en que contribuyen a la aparición de un mercado audiovisual europeo. 
Pese a ello, en el caso español la crisis económica ha tenido como consecuencia la recesión de un sector cinematográfico cuya quiebra ha llegado a pronosticarse.

Por el lado del consumo, los efectos de la depresión castigan evidentemente a la demanda, pero la subida brutal del IVA tiende a maximizar esos efectos. Pocos espacios quedan para imponer nuevas tasas solidarias al usuario, y la resistencia de las grandes empresas mediáticas a asumir compromisos estables a favor de la cultura dificulta toda solución. En cuanto a la oferta, el desplome de las políticas públicas está agudizando seriamente la quiebra de cientos de creadores y de PYMES, destruyendo una red de supervivencia que, aunque minoritaria en la financiación, constituía en muchos casos un sostén esencial (Bustamante, 2013, p. 39).

Sea como fuere, lo cierto es que el artículo 44.1 de la Constitución Española proclama que "los poderes públicos promoverán y tutelarán el acceso a la cultura, a la que todos tienen derecho”, lo que entra en contradicción con los recortes y políticas fiscales llevadas a cabo en los últimos tiempos. Aunque no se debe obviar el hecho de que los reales decretos de traspasos de funciones y servicios en materia cultural a las comunidades autónomas les otorgaron la competencia exclusiva en materia de espectáculos. Por lo que la variedad de posturas y modelos de financiación pública de la industria cinematográfica española se ha multiplicado desde entonces.

Durante años se ha venido proponiendo el modelo francés de apoyo al cine ${ }^{1}$ como ejemplo a seguir, pero lo cierto es que su aplicación en España se presume muy compleja. Del mismo modo, el mecenazgo de masas o crowdfunding impulsado ampliamente a través de Internet en los últimos años, tampoco parece ser la respuesta a los problemas del sector, pese a que el volumen de las transacciones en España se haya incrementado un 113\% con respecto a 2013, alcanzando los 62 millones de euros en 2014 (Wardrop, et al., 2015, p. 33).

Del nivel de compromiso de la autoridades estatales con la puesta en práctica de políticas y modelos que conduzcan a su solución da muestra, por ejemplo,

1. El Centre National de la Cinématographie et de l'image animée se financia a través de 
el hecho de que la cultura no fuera más que una Secretaría de Estado que comparte Ministerio con la educación (1996-2000) y el deporte (2000-2004) ya desde los gobiernos de José María Aznar. De hecho, tras las protestas por la subida del IVA cultural, las autoridades culturales no mostraron más que buena voluntad mediante la creación de mesas de trabajo sectoriales para la búsqueda de alternativas. Es más, pese a la promesa del ministro Ignacio Wert en el Senado sobre la creación de un nuevo sistema de ayudas al cine español antes de mayo de 2013, ha tenido que ser su sucesor en el cargo, el ministro Íñigo Méndez de Vigo, el que finalmente convocase en julio de 2015 a representantes del sector del cine y del audiovisual ${ }^{2}$ para informarles del cambio de modelo de financiación del cine aprobado mediante el Real Decreto-ley 6/2015, de 14 de mayo de $2015^{3}$.

Lo cierto es que los precedentes de las ayudas al cine arrancan en 1980, cuando se aprobaba la primera Ley del Cine de la democracia, que modificaba las cuotas de pantalla ya establecidas por el franquismo a un día de cine español por cada tres de película extranjera doblada. La sustituiría la Ley del Cine de 1983, la Ley Miró, que fijaba un sistema de subvenciones anticipadas de hasta el 50\% del coste de las películas, dinero que procedía del Fondo de Protección, y que otorgaba un comité que juzgaba su calidad previa, de forma que las ayudas se destinaban a películas que aún no habían comenzado a rodarse (subvenciones a priori). La llegada del Partido Popular al gobierno en 1996 redujo al mínimo las subvenciones concedidas a priori

2 En la reunión estuvieron presentes: Ramón Colóm, presidente de la Confederación de Asociaciones de Productores Audiovisuales de España (FAPAE); José Antonio Félez, presidente de la Asociación Estatal de Cine (AEC); Puy Oria, presidenta de la Asociación Madrileña Audiovisual (AMA); Estela Artacho, presidenta de la Federación de distribuidores cinematográficos (FEDICINE); Antonio Resines, presidente de la Academia del Cine; Juan Ramón Gómez Fabra, presidente de la Federación de Entidades de Empresarios de Cine de España (FECE); Mikel Lejarza, presidente de A3 Media Cine; Miguel Morales, presidente de la Asociación de distribuidores independientes cinematográficos (ADICINE); Carlos Biern, presidente de la Federación Española de Asociaciones de Productores de Animación (DIBOOS); Ghislain Barrois, consejero delegado de Telecinco Cinema; Fernando López Puig, director de Cine y Ficción de RTVE; además de José María Lassalle, secretario de Estado de Cultura; Fernando Benzo, subsecretario del Ministerio de Educación, Cultura y Deporte; Lorena González, directora general del Instituto de la Cinematografía y de las Artes Audiovisuales (ICAA) y José Canal, secretario general Técnico.

3 Real Decreto-ley 6/2015, de 14 de mayo, por el que se modifica la Ley 55/2007, de 28 de diciembre, del Cine, se conceden varios créditos extraordinarios y suplementos de créditos en el presupuesto del Estado y se adoptan otras medidas de carácter tributario. 
en beneficio de las ayudas a las películas que alcanzaban mayor recaudación en taquilla (subvenciones a posteriori). Por su parte, la Ley del Cine impulsada por la ministra González-Sinde en 2009, en vigor desde 2011, mantenía el sistema de ayudas a la amortización en función de lo recaudado en taquilla (ayudas a posteriori). La nueva Ley del Cine, las sustituye por ayudas anticipadas.

A lo anterior se añade la diversidad de discursos y modelos propuestos en función de su ideología por las autoridades autonómicas, regionales y municipales de turno, con lo cual "es complicado argumentar que en el Estado español existe una única política cultural o que haya una dirección unívoca” (Rowan, 2010, p. 40).

Así pues, la estructura del gasto público en cultura en España otorgaba tradicionalmente un papel menor al Estado central frente al realizado por las comunidades autónomas y la Administración municipal, apostando por la descentralización y las políticas culturales de proximidad auspiciadas por la Unión Europea. La consecuencia fue que el efecto sobre la cultura se multiplicó porque también fueron menores las transferencias del gobierno central a instituciones o actividades culturales de las comunidades autónomas, que soportaron un descenso del 42\% en sólo dos años (20112013). Un modelo que se ha mantenido hasta el año 2014, cuando por primera vez se incrementaron las ayudas de la Administración central (MECD, 2015, p. 27).

Y es que durante los últimos cinco años, mientras que el Ministerio de Educación, Cultura y Deporte reducía a la mitad sus ayudas al cine español, las comunidades autónomas hacían lo mismo. Esto, junto con el descenso de las inversiones en el sector cinematográfico por parte de las televisiones públicas y privadas, tras el hundimiento de sus ingresos publicitarios, comprometía la financiación de un sector al que apenas le quedaban alternativas. 


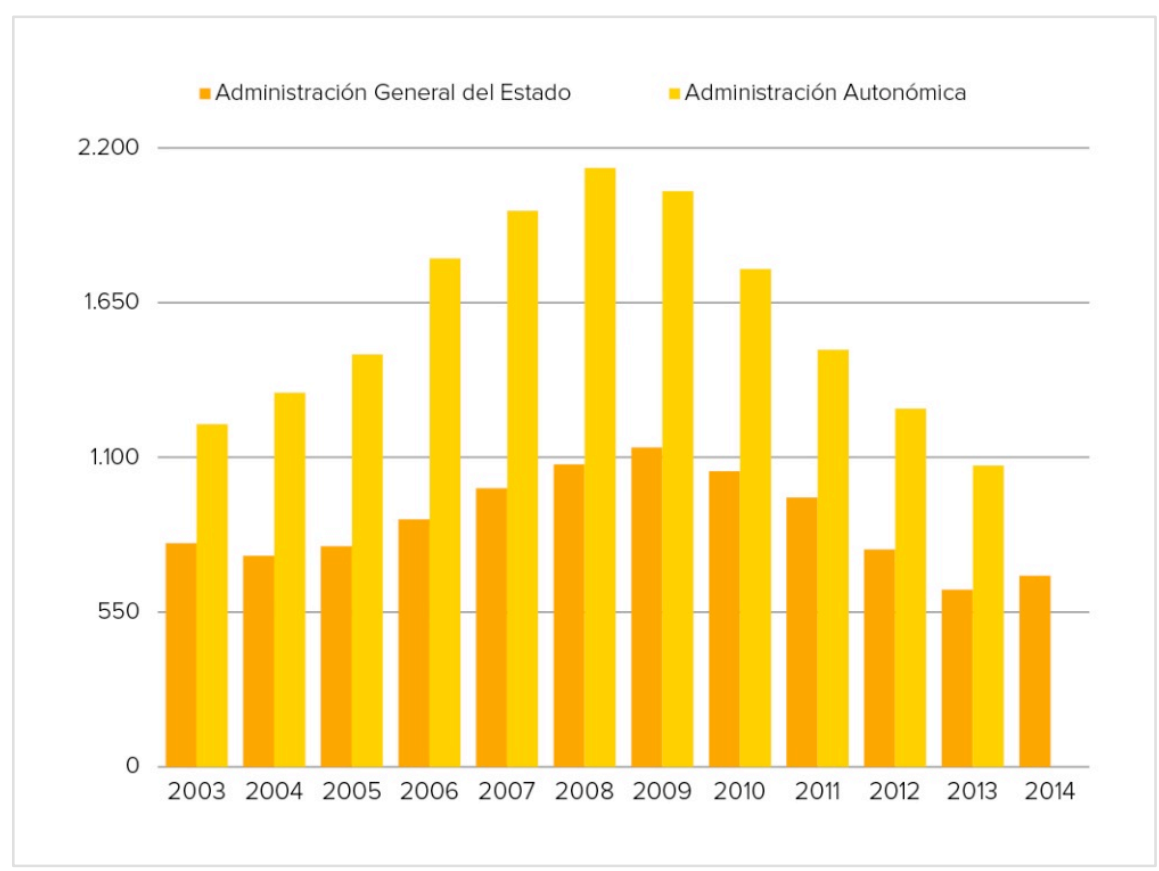

F2. Evolución del gasto liquidado en cultura por la Administración central del Estado y por la Administración autonómica en millones de euros. Anuario de Estadísticas Culturales 2015 (MECD, 2015, p. 27).

Así, yendo de lo general a lo particular y según los datos oficiales más recientes recogidos en el Anuario de Estadísticas Culturales 2015 a partir de la Estadística de Financiación y Gasto Público en Cultura elaborada por el Ministerio de Educación, Cultura y Deporte en colaboración con la Oficina Presupuestaria del Ministerio y con las comunidades autónomas, junto con las cifras de la Estadística de Liquidaciones de los Presupuestos de las Entidades Locales elaborada por el Ministerio de Hacienda y Administraciones Públicas, el gasto liquidado en cultura por las administraciones general, autonómica y local ha registrado descensos interanuales significativos desde 2009 (2015, p. 27).

No obstante, al respecto de la industria cinematográfica, los datos más recientes de los Presupuestos Generales del Estado de 2016 revelan un fuerte impulso a la tendencia iniciada en 2015, cuando por primera vez desde 2009 se incrementaba la inversión estatal en esta partida (74.539,15 millones de euros en 2016 frente a los 50.694,50 millones de euros de 2015). 


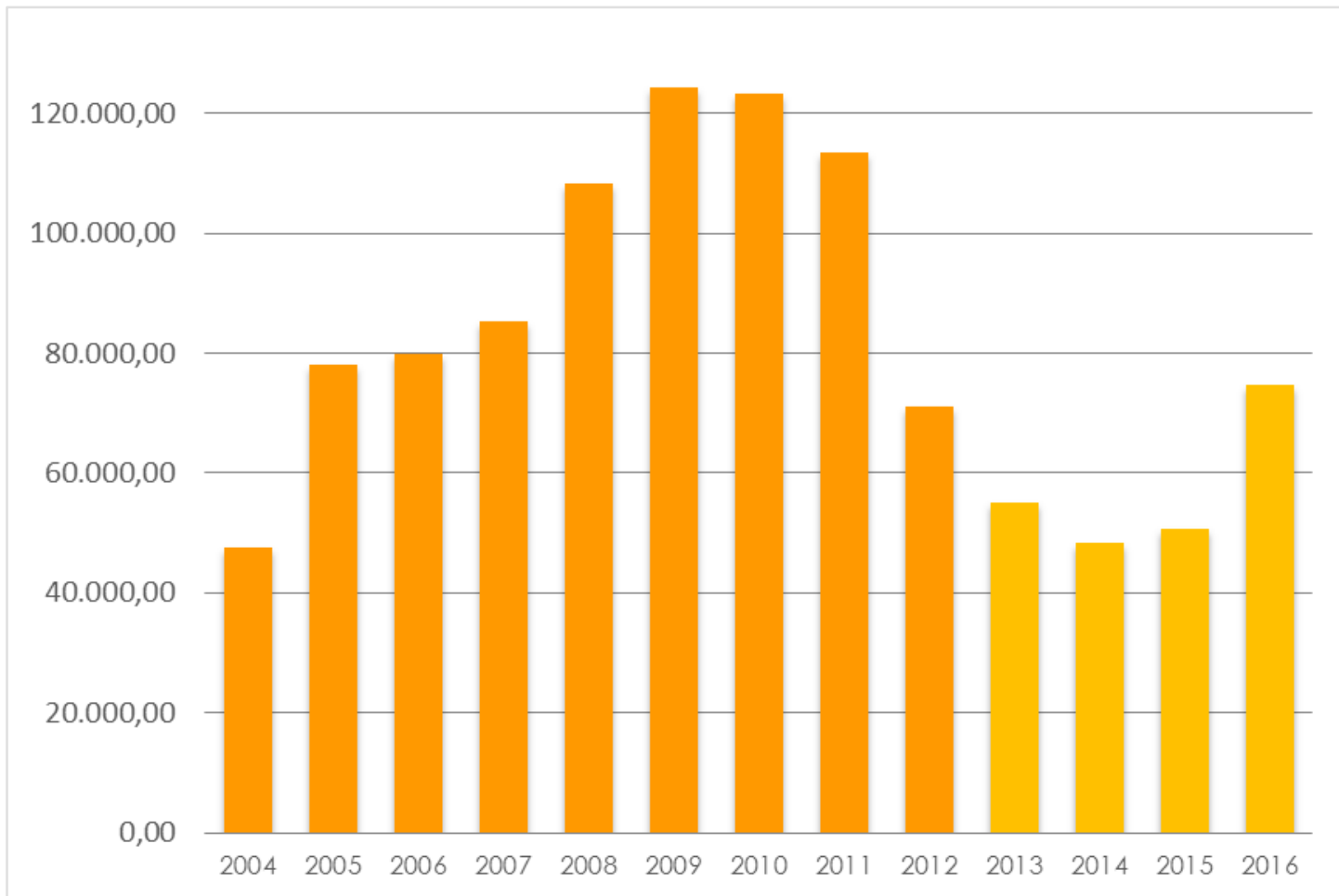

F3. Evolución de la dotación presupuestaria de la Administración del Estado a la Cinematografía (Programa 335C) en miles de euros. Elaboración propia a partir de los Presupuestos Generales del Estado.

Así, por ejemplo, en el caso concreto de las ayudas al sector de la exhibición cinematográfica, la nueva Ley del Cine, de reciente entrada en vigor, adapta el sistema de ayudas a la cinematografía y al audiovisual a la Comunicación de la Comisión sobre la Ayuda estatal a las obras cinematográficas y otras producciones del sector audiovisual (Diario Oficial de la Unión Europea de 15 de noviembre de 2013), principal instrumento normativo que actualmente ordena y limita los mecanismos excepcionales que la mayor parte de los países europeos han aprobado para apoyar la industria de su sector cinematográfico.

\begin{tabular}{llcccl}
\hline Año & $\begin{array}{c}\text { Presu- } \\
\text { puesto }\end{array}$ & $\begin{array}{c}\text { No de } \\
\text { solici- } \\
\text { tudes }\end{array}$ & $\begin{array}{c}\text { No de } \\
\text { ayudas } \\
\text { conce- } \\
\text { didas }\end{array}$ & Importe & \multicolumn{1}{c}{$\begin{array}{c}\text { Sala de exhibición } \\
\text { beneficiaria }\end{array}$} \\
\hline $\mathbf{2 0 0 6}$ & $500.000 €$ & 9 & 1 & $\mathbf{3 9 . 2 2 9 €}$ & $\begin{array}{l}\text { Compañía Inmobiliaria y de } \\
\text { Inversiones, S.A. }\end{array}$ \\
\hline $\mathbf{2 0 0 7}$ & $500.000 €$ & 2 & 1 & $\mathbf{3 2 . 0 0 0 €}$ & EXPINMOBEL, S.A. \\
\hline $\mathbf{2 0 0 8}$ & $500.000 €$ & 2 & 1 & $\mathbf{4 9 . 0 7 1 €}$ & Oscar Tarragona, S. L. \\
\hline
\end{tabular}




\begin{tabular}{|c|c|c|c|c|c|}
\hline \multirow[t]{7}{*}{2009} & $300.000 €$ & 7 & 6 & $73.763 €$ & \\
\hline & & & & $34.000 €$ & Cinemax Almenara (Lorca) \\
\hline & & & & $9.754,43 €$ & Cine Bages Centre (Manresa) \\
\hline & & & & $2.548,64 €$ & Cine ABC (Gandía) \\
\hline & & & & $9.609,68 €$ & Cine ABC (Elche y El Saler) \\
\hline & & & & $8.984,5 €$ & Cine Llobregat Centre (Cornella) \\
\hline & & & & $8.865,72 €$ & El Punt (Cendanyola del Valles) \\
\hline 2011 & $424.800 €$ & & & & \\
\hline \multirow[t]{5}{*}{2012} & $124.800 €$ & 5 & 4 & $16.558,64 €$ & \\
\hline & & & & $6.160 €$ & Cines ARCCA (Calahorra) \\
\hline & & & & $2.334,64 €$ & Cines Valleaguado \\
\hline & & & & $4.032 €$ & Multicines Tenerife \\
\hline & & & & $4.032 €$ & Multicines Tenerife \\
\hline \multirow[t]{6}{*}{2013} & $100.000 €$ & 5 & 5 & $25.454,14 €$ & \\
\hline & & & & $4.836,8 €$ & Cines El Castillo y Cines El Molino \\
\hline & & & & $3.200 €$ & Cine Velasco (Astorga) \\
\hline & & & & $7.040 €$ & Cines Babel \\
\hline & & & & $5.360 €$ & Cines Babel \\
\hline & & & & $5.017,34 €$ & Cine Olwyn Centre (Cambrils) \\
\hline \multirow[t]{3}{*}{2014} & 31.321 & 5 & 2 & $11.200 €$ & \\
\hline & & & & $3.200 €$ & Autocine de Torrelavega \\
\hline & & & & $8.000 €$ & Séptimo Oficio (Las Rozas) \\
\hline
\end{tabular}

F4. Resumen de las ayudas estatales a la minoración de intereses de préstamos bancarios para la digitalización de salas concedidas entre 2006 y 2014. Elaboración propia a partir de las Memorias de Ayudas a la Cinematografía del Instituto de la Cinematografía y de las Artes Audiovisuales (ICAA).

Tratan de revertir así la situación de los exhibidores, que forzados a la reconversión de las salas por la proximidad del apagón analógico se han visto obligados en muchos casos a cerrar los cines ante la imposibilidad de hacer frente a las deudas contraídas con las entidades de crédito. Y es que tanto las ayudas estatales como los convenios del Instituto de la Cinematografía y de las Artes Audiovisuales con el Instituto de Crédito Oficial (ICO) a través del programa ICO Empresas y Emprendedores, para apoyar las inversiones en digitalización de salas de exhibición cinematográfica de los años 2012, 2013, 20144, así como las ayudas para la minoración de intereses de los préstamos

4 En el año 2010 no se convocaron este tipo de ayudas. En 2011 estuvieron presupuestadas en 424.80o€, pero no hay datos sobre el número e importe de las ayudas concedidas. Las ayudas del ejercicio 2015 se convocaron en el BOE de 12 de septiembre de 2015, estando abierto el plazo de presentación de solicitudes hasta el 16 de octubre de 2015. La resolución 
concedidos a los exhibidores para el mismo fin, se han demostrado insuficientes.

La otra novedad incluida en la reforma de la Ley del Cine es la desaparición de las ventanas de exhibición (un período de demora obligatorio de tres meses entre el estreno en las salas y la comercialización en otros formatos de las películas perceptoras de ayudas públicas).

Así pues, el nuevo sistema de subvenciones se configura con las siguientes modalidades de ayudas y subvenciones: ayudas a la producción de largometrajes y cortometrajes, ayudas a la amortización de largometrajes (divididas en ayudas generales y complementarias), ayudas a la minoración de intereses de préstamos bancarios para la financiación de la producción de largometrajes, ayudas a la distribución de películas españolas, comunitarias e iberoamericanas, ayudas a la conservación de negativos y soportes originales y, por último, ayudas para la participación de películas españolas en festivales.

\begin{tabular}{|c|c|c|c|c|c|}
\hline \multicolumn{2}{|c|}{ Tipos de Ayudas } & 2013 & 2014 & 2015 & $\begin{array}{l}\text { \% varia- } \\
\text { ción } \\
\text { respecto } \\
\text { al año } \\
\text { anterior }\end{array}$ \\
\hline \multirow[t]{2}{*}{ Minoración } & $\begin{array}{l}\text { Exhibición cinematográfica y equi- } \\
\text { pamientos de producción cinema- } \\
\text { tográfica }\end{array}$ & $100.000 €$ & $31.321 €$ & $0 €$ & $-100 \%$ \\
\hline & Producción cinematográfica & $1.500 .000 €$ & $1.000 .000 €$ & $500.000 €$ & $-50 \%$ \\
\hline \multicolumn{2}{|c|}{ Producción de largometrajes sobre proyecto } & $5.000 .000 €$ & $4.000 .000 €$ & $4.750 .000 €^{*}$ & $18,75 \%$ \\
\hline \multicolumn{2}{|c|}{ Amortización de largometrajes } & $26.000 .000 €$ & $52.500 .000 €$ & $41.177 .068 €$ & $-21,57 \%$ \\
\hline \multicolumn{2}{|c|}{ Conservación del patrimonio cinematográfico } & $300.000 €$ & $300.000 €$ & $300.000 €$ & $0 \%$ \\
\hline \multicolumn{2}{|c|}{$\begin{array}{l}\text { Distribución de películas españolas, comunitarias } \\
\text { e iberoamericanas }\end{array}$} & $2.500 .000 €$ & $2.500 .000 €$ & $2.500 .000 €$ & $0 \%$ \\
\hline \multicolumn{2}{|c|}{$\begin{array}{l}\text { Ayudas para la participación y la promoción de } \\
\text { películas en festivales internacionales. }\end{array}$} & $500.000 €$ & $500.000 €$ & $300.000 €$ & $-40 \%$ \\
\hline \multicolumn{2}{|c|}{$\begin{array}{l}\text { Financiación de cortometrajes (ayuda sobre } \\
\text { proyecto y corto realizado) }\end{array}$} & $1.000 .000 €$ & $1.000 .000 €$ & $700.000 €$ & $-30 \%$ \\
\hline \multicolumn{2}{|l|}{ TOTAL } & $36.900 .000 \mathrm{C}$ & $61.831 .321 \mathrm{C}$ & $50.227 .068 \mathrm{C}$ & $-18,77 \%$ \\
\hline
\end{tabular}

F5. Comparación presupuestaria de las diferentes modalidades de ayudas a la cinematografía otorgadas por el ICAA entre 2013 y 2015. Memoria de Ayudas a la cinematografía 2015 (ICAA, 2016, p. 2).

sobre la cuantía y los beneficiados sigue pendiente de publicación. 


\subsection{Metodología}

Tras la aprobación en Consejo de Ministros en mayo de 2015 del Real Decreto-ley que modifica los parámetros para la financiación del cine español, implantando un nuevo modelo de subvenciones a priori en base a la viabilidad económica de los proyectos y a su potencialidad en lo que a la generación de espectadores se refiere, cabe plantearse si este nuevo sistema pondrá fin a la controversia existente sobre si las ayudas al cine tienen realmente efectos positivos en el sector del cine español.

En este sentido, el objetivo y finalidad de esta investigación se circunscribe a la determinación de la existencia de una correlación positiva entre las cuantías anuales de las ayudas estatales a la cinematografía y los principales indicadores del estado de la industria cinematográfica en España: número de películas españolas producidas, número de películas españolas estrenadas y total de espectadores del cine español.

En consecuencia, las hipótesis principales a verificar serían:

- Existe una correlación positiva entre la cuantía anual de las ayudas estatales a la cinematografía y el número de películas españolas producidas al año (hipótesis 1).

- Existe una correlación positiva entre la cuantía anual de las ayudas estatales a la cinematografía y el número de películas españolas estrenadas al año (hipótesis 2).

- Existe una correlación positiva entre la cuantía anual de las ayudas estatales a la cinematografía y el total de espectadores anuales del cine español (hipótesis 3).

Como hipótesis secundarias se formulan las siguientes:

- Existe una correlación positiva entre el número de películas españolas producidas al año y el número de películas españolas estrenadas al año (hipótesis 4).

- Existe una correlación positiva entre el número de películas españolas estrenadas al año y el total de espectadores anuales del cine español (hipótesis 5). 
Este planteamiento implica la cuantificación de cuatro variables durante un determinado período de tiempo: ayudas anuales a la cinematografía, películas españolas producidas anualmente, películas españolas estrenadas anualmente y número de espectadores anuales del cine español. Se trata de un estudio cuantitativo correlacional y diacrónico (o longitudinal) elaborado a partir de la dotación presupuestaria a la Cinematografía (programa 335C) recogida en los Presupuestos Generales del Estado para la cuantificación de la primera variable, y con los datos recogidos en el Boletín informativo del cine de 2014 del Instituto de la Cinematografía y de las Artes Audiovisuales (ICAA), relativos a las otras tres variables.

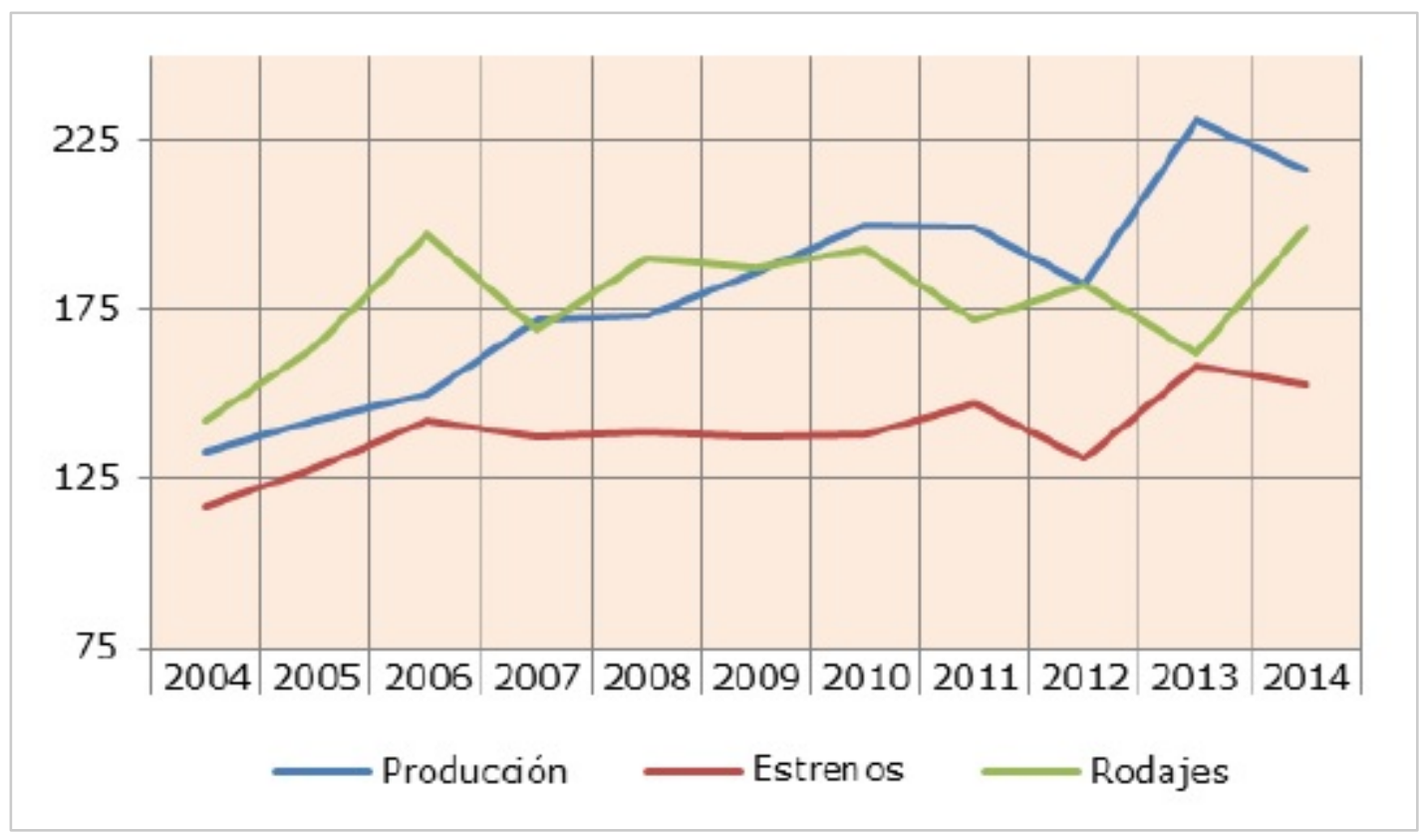

F6. Evolución de la producción, estrenos y rodajes de largometrajes españoles de 2004 a 2014. Boletín informativo del cine de 2014 (ICAA, 2015, p. 8).

El período de estudio se extiende entre los años 2004-2014, puesto que es el ámbito temporal sobre el que el Boletín informativo del cine de 2014, el último publicado, ofrece datos de tres de las cuatro variables analizadas. Además, dicho período coincide con el analizado por las tres últimas ediciones (2006-2007, 2010-2011, 2014-2015) de la Encuesta de Hábitos y Prácticas Culturales en España, una operación estadística desarrollada por el Ministerio de Educación, Cultura y Deporte e incluida en el Plan Estadístico Nacional. Se trata de una investigación dirigida a una muestra de 
16.0oo personas de 15 años en adelante residentes en viviendas familiares del territorio nacional y que ofrece información válida para contextualizar la evolución del consumo de cine español como elemento cultural. En consecuencia, se optó por circunscribir el análisis longitudinal de la correlación entre las cuatro variables estudiadas a once años, un periodo que por insuficiente podría mermar la representatividad del análisis, así como dificultar la extrapolación de los resultados y la obtención de conclusiones generalizables, una limitación que habrá de tenerse en cuenta a la hora de valorar las conclusiones en su justa medida.

Asimismo, el análisis estadístico de los datos para determinar la existencia de una correlación entre las cuatro variables estudiadas dos a dos, se estableció con la finalidad de responder a cuatro preguntas:

1. ¿Están relacionadas las variables entre sí? Si los cambios en el valor de una de las variables van acompañados de cambios en el valor de la otra, las variables parecen estar relacionadas.

2. Si las variables parecen estar relacionadas, ¿̇cuánto de intensa es la relación entre las variables?

3. ¿La relación entre las variables es positiva o negativa?

4. ¿Cuál es la relación causal entre las variables?

Para ello se optó por calcular el coeficiente de Pearson (r)5, cuya fórmula figura a continuación, y que da respuesta a tres de las cuatro cuestiones, indicando si dos variables parecen estar correlacionadas o no, la fuerza de la aparente relación y, por último, si la relación es positiva o negativa. No obstante, la relación causal entre cada par de variables estudiadas queda sin respuesta y, en este caso, como objeto de estudio de futuras investigaciones.

$$
\begin{gathered}
r=\frac{\sum x y}{\sqrt{\left(\sum x^{2}\right)\left(\sum y^{2}\right)}} \\
x=X-\bar{X} ; \quad y=Y-\bar{Y}
\end{gathered}
$$

5 También llamado coeficiente de correlación del producto-momento. 


\section{Exposición}

Tras la aplicación de la fórmula de la correlación de Pearson a los datos de las cuatros variables en el período estudiado (2004-2014), se obtiene el valor del coeficiente de correlación de Pearson, aunque hay que remarcar que éste no es el de la correlación per sé, entre:

- La cuantía anual de las ayudas estatales a la cinematografía y el número de películas españolas producidas al año.

- La cuantía anual de las ayudas estatales a la cinematografía y el número de películas españolas estrenadas al año.

- La cuantía anual de las ayudas estatales a la cinematografía y el total de espectadores anuales del cine español.

- El número de películas españolas producidas al año y el número de películas españolas estrenadas al año.

- El número de películas españolas producidas al año y el total de espectadores anuales del cine español.

- El número de películas españolas estrenadas al año y el total de espectadores anuales del cine español.

Los valores obtenidos fueron los siguientes:

\begin{tabular}{|l|r|r|r|r|}
\hline Años 2004 al 2014 & $\begin{array}{l}\text { Ayudas } \\
\text { amuales a la } \\
\text { cinematografía }\end{array}$ & $\begin{array}{l}\text { Películas } \\
\text { españolas } \\
\text { producidas } \\
\text { al año }\end{array}$ & $\begin{array}{l}\text { Películas } \\
\text { españolas } \\
\text { estrenadas } \\
\text { al año }\end{array}$ & $\begin{array}{l}\text { Total anual de } \\
\text { espectadores de } \\
\text { cine español }\end{array}$ \\
\hline $\begin{array}{l}\text { Ayudas anuales } \\
\text { ala } \\
\text { cinematografia }\end{array}$ & 1,000 & 0,077 & 0,008 & $-0,405$ \\
\hline $\begin{array}{l}\text { Películas } \\
\text { españolas } \\
\text { producidas } \\
\text { al año }\end{array}$ & 0,077 & 1,000 & 0,845 & $-0,494$ \\
\hline $\begin{array}{l}\text { Películas } \\
\text { españolas } \\
\text { estrenadas } \\
\text { al año }\end{array}$ & 0,008 & 0,845 & 1,000 & $-0,376$ \\
\hline $\begin{array}{l}\text { Total anual de } \\
\text { espectadores de } \\
\text { cine español }\end{array}$ & $-0,405$ & $-0,494$ & $-0,376$ & 1,000 \\
\hline
\end{tabular}

F7. Magnitudes del coeficiente de correlación de Pearson para el período y las cuatro variables estudiadas. Elaboración propia. 
Es importante tomar en consideración para el análisis de los datos que el coeficiente de correlación de Pearson (r) se mide en una escala de -1 a +1 . Así, un valor de $\mathrm{r}=\mathrm{o}$ revela que no existe relación lineal entre las variables, un valor de $\mathrm{r}=1$ indica una correlación perfecta positiva (al crecer una variable, crece la otra), mientras que un valor $\mathrm{r}=-1$ refleja una correlación perfecta negativa (al crecer o decrecer una variable, decrece o crece la otra). No obstante, lo más habitual es que el valor del coeficiente se sitúe en algún punto entre o $\mathrm{y}+1$ o entre $\mathrm{o} \mathrm{y}-1$.

Algo fundamental a tener en cuenta es que una correlación negativa no es menos fuerte que una correlación positiva, puesto que los signos positivos y negativos sólo indican si el valor de una variable aumenta o disminuye respectivamente con el aumento en el valor de la otra variable. Así, cuando el valor de la correlación es igual a $+10-1$ la covariación es de intensidad máxima, y se va acercando hacia el o cuanto más pequeña sea la intensidad de la covariación. Además, cuando los aumentos (disminuciones) de una variable producen aumentos (disminuciones) en la otra, la relación es positiva; es negativa cuando los aumentos (disminuciones) de una variable producen disminuciones (aumentos) en la otra. La magnitud de la correlación se ha interpretado siguiendo la siguiente escala:

\begin{tabular}{|c|l|}
\hline Valor & \multicolumn{1}{|c|}{ Significado } \\
\hline-1 & Correlación negativa perfecta \\
\hline$-\mathbf{0 , 9}$ a $-\mathbf{0 , 9 9}$ & Correlación negativa muy alta \\
\hline$-\mathbf{0 , 7}$ a $-\mathbf{0 , 8 9}$ & Correlación negativa alta \\
\hline$-\mathbf{0 , 4}$ a $-\mathbf{0 , 6 9}$ & Correlación negativa moderada \\
\hline$-\mathbf{0 , 2}$ a $-\mathbf{0 , 3 9}$ & Correlación negativa baja \\
\hline $\mathbf{- 0 , 0 1}$ a $-\mathbf{0 , 1 9}$ & Correlación negativa muy baja \\
\hline $\mathbf{0}$ & Correlación nula \\
\hline $\mathbf{0 , 0 1}$ a 0,19 & Correlación positiva muy baja \\
\hline $\mathbf{0 , 2}$ a 0,39 & Correlación positiva baja \\
\hline $\mathbf{0 , 4}$ a 0,69 & Correlación positiva moderada \\
\hline $\mathbf{0 , 7}$ a 0,89 & Correlación positiva alta \\
\hline $\mathbf{0 , 9}$ a 0,99 & Correlación positiva muy alta \\
\hline $\mathbf{1}$ & Correlación positiva perfecta \\
\hline & \\
\hline
\end{tabular}

F8. Escala de interpretación del coeficiente de correlación lineal de Pearson. Diseño y análisis de experimentos (Castejón, 2011, p. 90). 
El análisis de los resultados obtenidos muestra que existe una correlación positiva muy baja entre las ayudas anuales a la cinematografía y el número de películas españolas producidas al año $(\mathrm{r}=0,077)$, siendo nula la correlación entre las ayudas anuales a la cinematografía y el número de películas españolas estrenadas al año $(r=0,008)$.

Sin embargo, los datos analizados revelan que sí existe una correlación positiva alta entre el número de películas españolas producidas anualmente y el número de películas españolas estrenadas anualmente $(\mathrm{r}=0,845)$, que además coincide con lo deseado por todos los agentes del sector.

Asimismo, los valores obtenidos revelan la existencia de una correlación negativa moderada entre la cuantía de las ayudas anuales a la cinematografía y el total anual de espectadores del cine español $(r=-0,405)$, una correlación negativa moderada entre el número de películas españolas producidas al año y el total anual de espectadores del cine español $(r=-0,494)$ y una correlación negativa baja entre el número de películas españolas estrenadas al año y el total anual de espectadores del cine español $(r=-0,376)$.

Datos que vendrían a contradecir las teorías según las cuales el fortalecimiento de la industria cinematográfica española debe sustentarse en un sistema de subvenciones que contribuya a su financiación. Y es que la existencia de una muy baja correlación positiva entre las ayudas al cine y el número de películas producidas, unido a la nula existencia de correlación con el número de películas estrenadas, junto a la correlación negativa moderada entre la cuantía de las ayudas y el total anual de espectadores del cine español, obliga a reflexionar y preguntarse si tiene algún sentido esta política de subvenciones. Lo lógico sería pensar que un incremento en las ayudas aumentaría tanto el número de películas españolas producidas como las estrenadas anualmente, y que esto tendría su reflejo en un incremento del total de espectadores de cine español, pero si los resultados no lo demuestran, tal y como es el caso, cobran sentido las posturas que argumentan que si una película es exitosa no le harán falta las ayudas públicas, del mismo modo que si el público no quiere ir a ver un filme, ¿qué sentido tiene obligarle a pagarlo vía impuestos? 
Por eso, son muchos los productores que piden que las ayudas lleguen a través de otras vías, como se hace en otros países; fundamentalmente, mediante las exenciones de impuestos para las empresas que participan en el sector o bien a través del apoyo al mecenazgo privado. Un planteamiento en el que cobra sentido la reivindicación de una política fiscal acorde con la situación real del sector mediante la reducción del IVA al 4-5 \% para todas las actividades culturales (IVA cultural mínimo) y la implementación de deducciones y desgravaciones fiscales con prioridad para las donaciones de personas físicas como medida para la promoción del mecenazgo social, sobre todo del crowdfunding. Sin olvidar, por supuesto, la necesidad de reforzar la coordinación entre las políticas y ayudas al sector tanto estatales como autonómicas buscando su articulación sistemática.

\section{Conclusiones}

El análisis de los datos revela al respecto de las hipótesis principales y secundarias planteadas que solo dos se confirman como ciertas: la hipótesis 1, relativa a la existencia de una correlación positiva entre la cuantía anual de las ayudas estatales a la cinematografía y el número de películas españolas producidas al año, y la hipótesis 4 , que enunciaba la existencia de una correlación positiva entre el número de películas españolas producidas al año y el número de películas españolas estrenadas al año.

No obstante, en el caso de la hipótesis 1, la intensidad tan baja de la correlación positiva entre la cuantía anual de las ayudas estatales a la cinematografía y el número de películas españolas producidas al año, le resta casi todo el valor para su ratificación como sólido argumento en la defensa del sistema de subvenciones a la industria cinematográfica.

Asimismo, es preciso poner de manifiesto que el periodo objeto de análisis se ha caracterizado por importantes variaciones en el ciclo económico, que consecuentemente influyen en los valores de las variables analizadas, lo que podría explicar en parte lo excepcional y contradictorio de alguno de los datos obtenidos. Asimismo, lo reducido del ámbito temporal de la 
investigación implica que cada uno de los datos tenga gran peso, de forma que un cambio en uno solo de ellos tiene gran influencia en el valor final alcanzado por el índice de correlación al que su cálculo afecte, desvirtuando su posible interpretación.

Es por ello que como líneas de estudio futuras se propone el análisis de las causas que justifican la muy débil correlación positiva entre la cuantía de las ayudas anuales a la cinematografía y el número de películas españolas producidas anualmente, así como la no correlación con el número de películas estrenadas al año, pese a que los defensores del sistema de subvenciones argumenten su existencia. Pero sobre todo cabría plantearse el porqué de la moderada correlación negativa entre la cuantía anual de las subvenciones al cine español y el total anual de sus espectadores, un hallazgo que desvirtúa cualquier razonamiento a favor de las ayudas al cine como instrumento de estímulo de la rentabilidad la industria cinematográfica.

\section{Referencias bibliográficas}

Álvarez, J. M. y López, J. (2016). La producción audiovisual: promesas a través de la crisis. En E. Bustamante (Coord.), Informe sobre el estado de la cultura en España. La cultura como motor de cambio 2016 (pp. 43-52). Madrid, España: Fundación Alternativas. http://www.fundacionalternativas.org/public/storage/publicaciones_ archivos/f826abeaa553a2cac49bb8d38c11dae3.pdf

Aranzubía, A. (2014). En busca de una política activa. Exhibición digital y diversidad cultural. Caimán Cuadernos de cine, 25, 82-83.

Asociación para la investigación de medios de comunicación (2016). Oleada de abril de 2015 a marzo de 2016 del Estudio General de Medios (EGM). Recuperado de http://www.aimc.es/-Datos-EGM-ResumenGeneral-.html

Asociación para la investigación de medios de comunicación (2016). Decimonoveno censo de salas de cine. Recuperado de http://www.aimc.es/-Censo-Cine-.html

Bustamante, E. (2013). España: La cultura en tiempos de crisis. Fuentes financieras y políticas públicas. Madrid, España: Fundación Alternativas. Recuperado de http://www.fomecc.org/recursos/67.pdf

Castejón, O. (2011). Diseño y análisis de experimentos. Maracaibo, Venezuela: Fondo Editorial Biblioteca Universidad Rafael Urdaneta. 
Comisión Europea (2013). Comunicación de la Comisión sobre la Ayuda estatal a las obras cinematográficas y otras producciones del sector audiovisual. Diario Oficial de la Unión Europea de 15 de noviembre de 2013. Recuperado de http://eur-lex.europa.eu/legalcontent/ES/TXT/PDF/?uri=OJ:C:2013:332:FULL\&from=ES

Comisión Europea (2014). Programa Europa Creativa. Cultura y medios audiovisuales. Luxemburgo: Oficina de publicaciones de la Unión Europea. Recuperado de http://europa.eu/pol/pdf/flipbook/es/culture_audiovisual_es.pdf DOI:10.2775/12332

Consejo de Europa (2001). Resolución del Consejo Europa sobre las ayudas nacionales al sector del cine y al sector audiovisual. Diario Oficial $C$ 73 de 6 de marzo de 2001. Recuperado de http://eurlex.europa.eu/legalcontent/ES/TXT/HTML/?uri=URISERV:124221a\&qid=14376820644 23\&from $=\mathrm{ES}$

García, J. V. (2015). La exhibición cinematográfica en España. Cincuenta años de cambios. Madrid: Cátedra.

Gobierno de España (2007). Ley del Cine: Ley 55/2007, de 28 de diciembre, del Cine. Boletín Oficial del Estado de 29 de diciembre de 2007. Recuperado de http://www.boe.es/buscar/pdf/2007/BOE-A-200722439-consolidado.pdf

Gobierno de España (2015). Real Decreto-ley 6/2015, de 14 de mayo, por el que se modifica la Ley 55/2007, de 28 de diciembre, del Cine. Boletín Oficial del Estado de 15 de mayo de 2015. Recuperado de http://www.boe.es/boe/dias/2015/05/15/pdfs/BOE-A-2015-5368.pdf

Instituto de la Cinematografía y de las Artes Audiovisuales. Secretaría de Estado de Cultura (2015). Boletín informativo del cine de 2014. Recuperado de http://www.mecd.gob.es/cultura$\mathrm{mecd} / \mathrm{dms} / \mathrm{mecd} /$ cultura-mecd/areas-cultura/cine/mc/anuariocine/ano-2014/Boletin-2014.pdf

Instituto de la Cinematografía y de las Artes Audiovisuales. Secretaría de Estado de Cultura (2016). Memoria de Ayudas a la cinematografía 2015. Recuperado de http://www.mecd.gob.es/culturamecd/dms/mecd/cultura-mecd/areas-cultura/cine/mc/mac/2015/1presentacion-presupuesto.pdf

Ministerio de Educación, Cultura y Deporte (2015). Anuario de Estadísticas Culturales 2015. Recuperado de http://www.mecd.gob.es/servicios-alciudadano-mecd $/ \mathrm{dms} / \mathrm{mecd} /$ servicios-al-ciudadanomecd/estadisticas/cultura/mc/naec/2015/Anuario_de_Estadisticas_ Culturales_2015.pdf

Ministerio de Educación, Cultura y Deporte (2015). El ministro de Educación, Cultura y Deporte se reúne con representantes del sector del Cine $y$ el Audiovisual. Recuperado de 
http://www.mecd.gob.es/prensamecd/actualidad/2015/07/20150707-cine.html

Ministerio de Educación, Cultura y Deporte (2015). Estadística de Hábitos y Prácticas Culturales en España 2014-2015. Recuperado de http://www.mecd.gob.es/mecd/dms/mecd/servicios-al-ciudadanomecd/estadisticas/cultura/mc/ehc/20142015/Encuesta_de_Habitos_y_Practicas_Culturales_2014-2015.pdf

Ministerio del Interior (2013). Comunidades autónomas con competencia en materia de espectáculos. Recuperado de http://www.interior.gob.es/web/servicios-alciudadano/espectaculos/espectaculos/comunidades-autonomas-concompetencia-en-materia-

Rentrak Spain (2016). Informe Anual 2015 Cine. Recuperado de http://usg.campaignarchive2.com/?u=da7543059f5e71fd8ac244f2e\&id=4da6a18afa

Rowan, J. (2010). Emprendizajes en cultura. Discursos, instituciones y contradicciones en la empresarialidad cultural. Madrid, España: Traficantes de Sueños.

Sociedad General de Autores y Editores (2016). Anuario de la SGAE de las artes escénicas, musicales y audiovisuales 2015. Recuperado de http://www.anuariossgae.com/anuario2015/frames.html

Subdirección General de Fomento de la Industria Cinematográfica y Audiovisual del Instituto de Cinematografía y de las Artes Audiovisuales (2014). Estadística Cinematográfica: Producción, Exhibición, Distribución y Fomento - avance 2014. Recuperado de http://www.mcu.es/culturabase/cgi/um?M=/t20/p20

Wardrop, R., Zhang, B., Rau, R. y Gray, M. (2015). The European Alternative Finance Benchmarking Report. Cambridge, United Kingdom: University of Cambridge. Recuperado de http://www.jbs.cam.ac.uk/index.php?id=6481\#.VcOMe_nj9ME

Cómo citar: Heredero Díaz, O. y Reyes Sánchez, F. (2017). Presente y futuro de las subvenciones a la industria cinematográfica española. Fotocinema. Revista científica de cine y fotografía, $\mathrm{n}^{\mathrm{o}}$ 14, pp. 341-363. Disponible: http://www.revistafotocinema.com/ 